\title{
Arthroscopic Bankart repair for first time versus recurrent anterior shoulder instability in athletes- comparative Study
}

S.A.Khalil, M.G.Montaser, M.S. Singer and A.H.Hassanin

Orthopedic Surgery, Dept., Faculty of Medicine, Benha Univ., Benha, Egypt

E-mail: Hassanin@yahoo.com

\begin{abstract}
Background: Following an initial traumatic anterior shoulder dislocation, the incidence of recurrent instability ranges from $14 \%$ to $100 \%$. The risk of recurrent dislocations is influenced by the age at the time of initial dislocation. The aim of the present study was to evaluate the results of arthroscopic bankart repair of acute anterior shoulder dislocation for first time in athletes and comparison the results with delayed arthroscopic bankart repair Patient and methods: The present study included 40 patients complaining of anterior shoulder dislocation, 20 patients of them were with first time anterior shoulder dislocation and 20 patients with recurrent dislocation. All patients underwent Arthroscopic Bankart Repair for the Management of Anterior Shoulder Instability. The constant shoulder score was used for assess the outcomes Results: There was statistically significant difference between pre and post-operative outcome in first time dislocation patients. There was statistically significant difference between pre and post-operative outcome in recurrent dislocation patients. There was no statistically significant difference according to post-operative outcomes between in first time and recurrent dislocation patients. Conclusion: there were no differences in outcomes and constant shoulder score among patients undergoing surgery after the primary dislocation when compared with those undergoing surgery after recurrent shoulder dislocation.
\end{abstract}

Keywords: Arthroscopic Bankart repair, recurrent anterior shoulder instability, The constant shoulder score.

\section{Introduction}

Traumatic anterior shoulder dislocations are a relatively common injury, with an incidence rate near 24 per 100000 person-years. The injury is 2 to 5 times more common in males, with almost half occurring before the age of 30. [1]

The stability of the glenohumeral joint is maintained by the glenoid labrum. This labrum creates a socket-deepening effect, hence preventing shoulder dislocation. The anteroinferior labrum also serves as the anchor point for the inferior glenohumeral ligament, the primary static restraint to the anterior humeral translation in the abducted shoulder. [2] An avulsion of the labrum from the glenoid rim is known as a Bankart lesion. [3]

Following an initial traumatic anterior shoulder dislocation, the incidence of recurrent instability ranges from $14 \%$ to $100 \%$. The risk of recurrent dislocations is influenced by the age at the time of initial dislocation. In patients less than twenty years old the rate of recurrent instability is $72-100 \%$, in those aged between $20-30$ years it is $70-82 \%$ and in patients more than fifty years old it is $14-22 \%$. [4]

Robinson et al found that arthroscopic repair of a Bankart lesion after primary traumatic anterior shoulder dislocation reduced the risk of recurrence by $76 \%$; they also found that the risk of all recurrent instability was reduced by $82 \%$ in this group of patients that underwent Bankart repair, as compared to the group that underwent arthroscopy and lavage alone because of the high rate of recurrence in this population of patients, many authors have begun advocating early surgical intervention for these "atrisk" patients. [5]

Lau et al. discovered a staggering preponderance of off-track Hill-Sachs lesions in adolescents with anterior dislocations in comparison to adults $(24.4 \%$ incidence in adolescents and only a $3.3 \%$ incidence in adults). Furthermore, they found that adolescence and a history of multiple dislocations are independent risk factors for having off-track Hill-Sachs lesions. These findings alone make a compelling case for doing arthroscopic Bankart repairs in adolescent first-time dislocators, before they develop off-track Hill-Sachs lesions that demand more extensive surgical interventions such as remplissage or even Latarjet reconstruction. [6]

Advocates for early intervention contend that these patients benefit from surgery because of a dramatically lower recurrence rate when compared with conservative measures. One may also deduce that early intervention allows for a more anatomic repair with "good" tissue, whereas patients with multiple episodes of instability may have attenuation of the anterior structures, making repair more difficult and tenuous at the time of surgery. Finally, authors argue that patients may have an improved quality of life with a more rapid return to preinjury activity level and a lower risk of recurrent episodes. [7]

The aim of the present study was to evaluate the results of arthroscopic bankart repair of acute anterior shoulder dislocation for first time in athletes and comparison the results with delayed arthroscopic bankart repair.

\section{Patient and Method}

This was prospective case study to be done at Benha university hospitals on 20 patients with first time anterior shoulder dislocation and 20 patients with recurrent dislocation. Teenager above 16years athletics highly active patients was included in this study. 


\subsection{Surgical Techniques}

Preoperative Planning Obtaining a thorough history is the first step in evaluating a patient with shoulder instability. It is important to inquire about the mechanism of injury, the direction of the instability, and the method of reduction.

A $30^{\circ}$ arthroscopic tissue liberator is used to elevate and free up the labrum from the anterior glenoid, which is often scarred down in a nonanatomic, medialized position this is an important step as incomplete mobilization of the anterior labrum from the glenoid neck may result in a nonanatomic, less functional repair. The subscapularis muscle can be seen anteriorly when this tissue is sufficiently elevated off the anterior glenoid neck. An arthroscopic rasp (Arthrex) is used to prepare the anterior capsular tissue for better healing after plication ,A small 3.5-mm burr (Smith \& Nephew) is then used to create a bleeding bed of bone along the neck of the glenoid This completes glenoid and soft tissue preparation

While viewing from the posterior portal, the drill guide for the suture anchor ( FASTak suture anchor is a fully threaded $2.4 \times 7.5 \mathrm{~mm}$ titanium, and suture Tac biocomposit $3 \mathrm{~mm} \times 14.5 \mathrm{~mm}$, Arthrex) is introduced through the inferior cannula and is positioned onto the face of the glenoid as close to the 6 o'clock position as possible The stout metal drill guide can be used to lever the humeral head slightly out of the way to get the best possible angle in the glenoid to prevent skiving and cartilage injury. If the most inferior position on the glenoid cannot be reached from the anterior cannula, a percutaneous portal through the subscapularis tendon can be used. A drill is used to create a pilot hole, and the anchor is then tapped into the glenoid. A suture-retrieving grasper (Arthrex) is used to grasp one limb of suture from the anchor and pull it out through the 5-mm cannula. A curved suture passer (Spectrum, ConMed, Utica, NY) loaded with a no. 1 polydioxanone (PDS) suture is used to pierce the capsule and is advanced under the capsule and labrum in 1 pass The goal is to get a healthy capsular bite, grabbing inferior to the anchor in an effort to shift the tissue superiorly, reducing capsular volume. The PDS is advanced out of the passer and into the joint, where it is grasped and pulled out of the $5 \mathrm{~mm}$ cannula. The suture passer is removed from the shoulder. A simple loop is then made with the PDS that was pulled out of the 5-mm cannula. This loop is loaded with the anchor suture and cinched down. The PDS limb that is in the 8-mm cannula is pulled, shuttling the suture limb through the tissue and out of the cannula.

Knots are tied arthroscopically using low-profile sliding locking Westin knots followed by half hitches with care to keep the knots away from the glenoid face, in an effort to prevent articular cartilage injury from the suture, Suture tails are cut with the arthroscopic cutters, leaving a small 2-mm tail. Subsequent anchors are placed in similar fashion, working superiorly on the glenoid. Anchors are placed as close to each other as possible without compromising fixation of the previous anchor. This recreates a stabilizing "bumper" effect on the anterior glenoid and ensures multiple points of fixation

\subsection{Postoperative Rehabilitation}

A shoulder immobilizer is worn for 6 weeks. Phase 1 (weeks 0-6) goals include protecting the anatomic repair, preventing the negative effects of immobilization, promoting dynamic stability and proprioception, and diminishing pain and inflammation. Passive and gentle active assistive ranges of motion (ROM) exercises are begun, but no active external rotation, extension, or abduction is allowed. During weeks 5 to 6 , the patient works to gradually improve ROM with gentle stretching exercises. Phase 2 (weeks 7-14) goals include working to gradually restore full ROM (ideally by week 10), restoring muscular strength and balance, and enhancing neuromuscular control. More aggressive strengthening and ROM are progressed to meet functional demands (i.e., overhead athlete). Phase 3 (weeks 15-20) goals include improving muscular strength, power, and endurance and gradually initiating functional activities. Next, the advanced strengthening phase (weeks 21-24) goals include enhancing muscular strength, power, and endurance, progressing functional activities, and maintaining shoulder mobility. Lastly, the return to activity phase (months 7-9) goals include a gradual return to sport activities while maintaining strength, mobility, and stability. The protocol may be modified slightly based on the athlete's goals, revision versus primary cases, and the tissue quality at the time of surgery.

\subsection{Postoperative evaluation protocol Clinical and functional evaluation:}

Absence of postoperative sublaxation or recurrent dislocation at short term follow up (2 years) and are performed using the constant shoulder score which depend on pain, activity level, arm positioning, strength and range of motion.

The basis of is a 100 point score composed of Pain is allocated 15 of the total 1 Scoring for individual parameters:

$\begin{array}{ll}\text { Pain } & 15 \\ \text { Activity of daily living } & 20 \\ \text { Range of motion } & 40 \\ \text { Power } & 25 \\ \text { Total } & 100\end{array}$

\subsection{Statistical Analysis}

The collected data was revised, coded, tabulated and introduced to a PC using Statistical package for Social Science (IBM Corp. Released 2011. IBM SPSS Statistics for Windows, Version 20.0. Armonk, NY: IBM Corp.). Data were presented and suitable analysis was done according to the type of data obtained for each parameter. 


\section{Results}

The mean age was $27.05 \pm 5.5$ years in first time anterior shoulder dislocation patients and $27.1 \pm 5.05$ years in patients with recurrent dislocation and all patients in both groups were males except one female in recurrent shoulder dislocation group. There was no statistically significant difference between the two groups according to demographic and anthropometric data.

The mean number of anchors was $2.7 \pm 0.55$ in First time shoulder dislocation patients and $2.95 \pm 0.49$ in recurrent shoulder dislocation patients. Follow up duration in months was $18.35 \pm 3.66 \mathrm{~m}$ in First time shoulder dislocation patients and $19.4 \pm 2.97 \mathrm{~m}$ in recurrent shoulder dislocation patients. Type of anchors was knotted in all cases in both groups.

According to type of sport, about half of patients in both groups played soccer and the rest of them played athletics, judo, wrestling, basketball and just trainers with no statistically significant difference between both groups

According to constant shoulder score between pre and post-operative in first time dislocation patients we obtained that pain, stability, Range of motion and function was significantly higher after surgery $(\mathrm{p}<0.0001)$
According to constant shoulder score between pre and post-operative in recurrent dislocation patients we obtained that pain, stability, range of motion, function $(\mathrm{p}<0.0001)$ and range of motion $(\mathrm{p}<0.029)$ was significantly higher after surgery

There was no statistically significant difference according to post-operative constant shoulder score between in first time and recurrent dislocation patients,

There was statistically significant difference between pre and post-operative outcome in first time dislocation patients

There was statistically significant difference between pre and post-operative outcome in recurrent dislocation patients

There was no statistically significant difference according to post-operative outcomes between in first time and recurrent dislocation patients.

According to complications, superficial complication was reported in 2 cases of first time shoulder dislocation group and 3 cases in recurrent shoulder dislocation group which was treated by local and systemic antibiotics. Postoperative stiffness was reported in 4 cases of first time shoulder dislocation group and 3 cases in recurrent shoulder dislocation group and managed by physiotherapy. One case in first time shoulder dislocation group showed recurrent shoulder dislocation at the period of follow up.

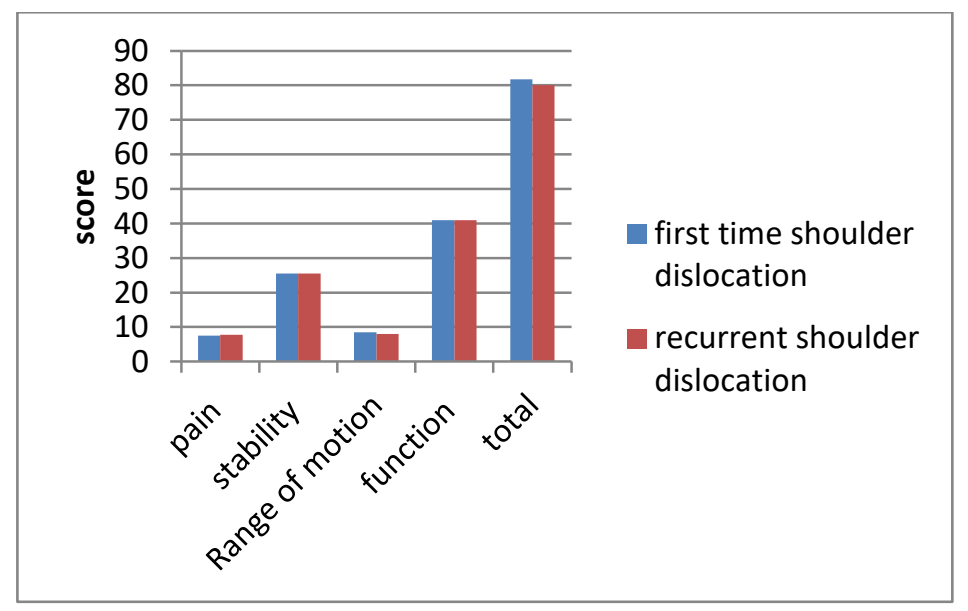

Fig. (1) Comparison post-operative constant shoulder score between in first time and recurrent dislocation patients

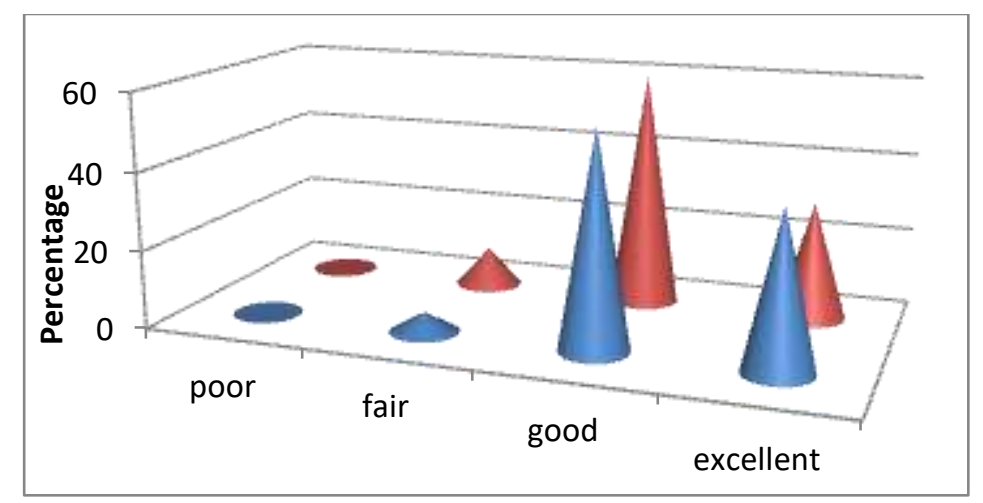

Fig. (2) Comparison post-operative outcomes between first time and recurrent dislocation patients 


\section{Discussion}

Recurrent anterior shoulder dislocation is one of the most common injuries in athletes. [8] Surgical treatment is generally recommended, especially in young patients due to the high redislocation rate after nonoperative treatment. [9]. Reports on open Bankart repair have shown high success rates in the long term, with redislocation rates that may be less than $5 \%$. [10, 11]

Twelve studies were included in [12] metaanalysis with a total of 799 shoulders, 518 in the primary dislocation group, 121 in the recurrent dislocation group, and 165 in the conservative group. The average age was 21.3 years, with 404 males and 54 females. The mean follow-up was 39.2 months.

The mean number of anchors in the present study was $2.7 \pm 0.55$ in First time shoulder dislocation patients and $2.95 \pm 0.49$ in recurrent shoulder dislocation patients. Follow up duration in months was $18.35 \pm 3.66 \mathrm{~m}$ in First time shoulder dislocation patients and $19.4 \pm 2.97 \mathrm{~m}$ in recurrent shoulder dislocation patients. Type of trauma was significant knotted in all cases in both groups and type of anchors was knotted in all cases in both groups.

The Follow up duration in months in [13] study was $39 \pm 15.3$ months for both groups. While in In [14] study, the mean follow-up in months in FirstTime $(\mathrm{n}=101)$ was $50.79 \pm 29.68$ and $51.1 \pm 31.22$ in Recurrent $(\mathrm{n}=72)$. The mean follow-up was 39.2 months in [12] meta-analysis

In the present study, according to constant shoulder score between pre and post-operative in first time dislocation patients we obtained that pain, stability, Range of motion and function was significantly higher after surgery $(p<0.0001)$. according to constant shoulder score between pre and post-operative in recurrent dislocation patients we obtained that pain, stability, function $(\mathrm{p}<0.0001)$ and range of motion $(p<0.029)$ was significantly higher after surgery

On the basis of the collection of studies gathered in [12] meta-analysis, there is no apparent difference in overall recurrence or complication rate among patients undergoing surgery after the first dislocation compared with those repaired after recurrent dislocation.

More specifically, when comparing the type of arthroscopic procedure performed, including modern techniques of suture anchor fixation, there is no difference in recurrence. Rates after arthroscopic suture anchor fixation approach those of open techniques. With regard to functional outcome, there was significant improvement in outcome from preoperative to postoperative assessment among independent studies. [15]

There was no statistically significant difference in the present study, according to post-operative constant shoulder score between in first time and recurrent dislocation patients. There was statistically significant difference between pre and post-operative outcome in first time dislocation patients. There was statistically significant difference between pre and post-operative outcome in recurrent dislocation patients. There was no statistically significant difference according to postoperative outcomes between in first time and recurrent dislocation patients,

The effect of multiple preoperative dislocations on arthroscopic Bankart repair outcomes has not been well described. [16] conducted a systematic review to evaluate recurrence rate, functional outcome, and quality of life among patients undergoing surgery after a primary dislocation and compared that with outcomes of those undergoing surgery after multiple dislocations. Several studies that met inclusion criteria were reviewed that classified patients as either with first-time or recurrent dislocations and compared reported outcomes. Clinical outcome measures significantly improved across all groups of patients postoperatively, and the conclusion was that there were no differences in recurrence or complication rate between patient groups.

\section{Conclusion}

There were no differences in outcomes and constant shoulder score among patients undergoing surgery after the primary dislocation when compared with those undergoing surgery after recurrent shoulder dislocation. Clinical outcome measures significantly improved within all independent studies from preoperatively to postoperatively. However, because of variation in the outcome measurement tools used, no direct comparison between the study groups could be performed. Additional randomized controlled studies are needed to compare the functional outcome, quality of life, and ability to return to preinjury activity level among patients undergoing early versus delayed repair for anterior shoulder instability.

\section{References}

[1] MD.Derek Smith, C.FRCS. First-time traumatic anterior shoulder dislocations in young patients BCMJ, Vol. 58, NSeptember,pp. 399-400, 2016.

[2] SJ.Turkel, MW.Panio, JL.Marshall, FG.Girgis. Stabilizing mechanisms preventing anterior dislocation of the glenohumeral joint. J Bone Joint Surg Am.vol.63,pp.1208-17,1981.

[3] CR.Rowe, D.Patel, WW.Southmayd. The Bankart procedure: a long-term end-result study. J Bone Joint Surg Am.vol.60,pp.116,1978 .

[4] D.Guillaume, D.Robert, J.William. Anterior shoulder instability: a review of pathoanatomy,diagnosis and treatment.Curr Rev Musculoskelet Med Streubel P,Krych A,Simone J,et al.Anterior Glenohumeral Instability: A Pathology.vol.4,pp.207,2011. ).

[5] CM.Robinson, PJ.Jenkins, TO.White, A.Ker, E.Will. Primary arthroscopic stabilization for a first-time anterior dislocation of the shoulder. A 
randomized, double-blind trial. J Bone Joint Surg Am.vol.90,pp.708-21,2008.

[6] B.C.Lau, D.Conway, P.F.Curran, B.T.Feeley, and N.K.Pandya. Bipolar bone loss in patients with anterior shoulder dislocation: A comparison of adolescents versus adult patients. Arthroscopy.vol.33,pp. 1755-1761,2017.

[7] M.Florian Buck , J.Tobias Dietrich, Donald Resnick, Bernhard Jost, W.A.Christian. Pfirrmann; MUSCULOSKELETAL IMAGING: Normal Position, Shape, and Orientation of Long Biceps Tendon. Radiology.vol. 261.pp.872-881,2011.

[8] BD.Owens, TM.De Berardino, BJ.Nelson. (Long-term follow-up of acute arthroscopic Bankart repair for initial anterior shoulder dislocations in young athletes. Am J Sports Med.vol.37,pp. 669-673,2009.

[9] BW.Jakobsen, HV.Johannsen, P.Suder, JO.Søjbjerg. Primary repair versus conservative treatment of first-time traumatic anterior dislocation of the shoulder: a randomized study with 10-year follow-up. Arthroscopy .vol.23(2),pp.118-123,2007.

[10] JD.Harris, AK.Gupta, NA.Mall. Long-term outcomes after Bankart shoulder stabilization. Arthroscopy .vol.29(5),pp.920-933,2013.

[11]TR.Lenters, AK.Franta, FM.Wolf, SS.Leopold, FA.Matsen. Arthroscopic compared with open repairs for recurrent anterior shoulder instability: a systematic review and meta- analysis of the literature. J Bone Joint Surg Am.vol.89(2),pp.244-254,2007.

[12] Adam, Mohammed \& Attia, Ahmed \& Alhammoud, Abduljabbar \& Aldahamsheh, Osama \& Dosari, Mohammed \& Alhaneedi, Ghalib. Arthroscopic Bankart repair for the acute anterior shoulder dislocation: systematic review and meta-analysis. International Orthopaedics.vol.8,pp.44-66 42,2018.

[13]M.S.Virk, R.L.Manzo, M.Cote, J.K.Ware, A.D.Mazzocca, C.W.Nissen, K.P.Shea, R.A. Arciero. Comparison of Time to Recurrence of Instability After Open and Arthroscopic Bankart Repair Techniques. Orthopaedic journal of sports medicine .vol.4(6), pp. $2325967116654114,2016$.

[14] T.Marshall, J.Vega, M.Siqueira, R.Cagle, J.D.Gelber, P.Saluan. Outcomes After Arthroscopic Bankart Repair: Patients With First-Time Versus Recurrent Dislocations. The American Journal of Sports Medicine.vol. 45(8),pp. 1776-1782,2017.

[15]CM.Robinson, PJ.Jenkins, TO.White, A.Ker, E.Will. Primary arthroscopic stabilization for a first-time anterior dislocation of the shoulder. A randomized, double-blind trial. J Bone Joint Surg Am.vol.90,pp.708-721,2008.

[16] R.Grumet, B.Bach, M.Provencher. Arthroscopic stabilization for first-time versus recurrent shoulder instability. Arthroscopy.vol.26(2),pp.239-248,2010. 